\title{
Effects of environment on stellar metallicity profiles of late-type galaxies in the CALIFA survey
}

\author{
Valeria Coenda ${ }^{1,2}$, Damián Mast ${ }^{2,3}$, Hernán Muriel ${ }^{1,2}$, and Héctor J. Martínez ${ }^{1,2}$ \\ ${ }^{1}$ Instituto de Astronomía Teórica y Experimental (IATE), CONICET - UNC, Laprida 854, X5000BGR Córdoba, Argentina \\ e-mail: vcoenda@unc.edu.ar \\ 2 Observatorio Astronómico, Universidad Nacional de Córdoba, Laprida 854, X5000BGR Córdoba, Argentina \\ ${ }^{3}$ Consejo de Investigaciones Científicas y Técnicas de la República Argentina, Avda. Rivadavia 1917, C1033AAJ CABA, Argentina
}

Received 27 June 2020 / Accepted 12 August 2020

\begin{abstract}
Aims. We explore the effects of environment in the evolution of late-type galaxies by studying the radial profiles of light- and massweighted metallicities of galaxies in two discrete environments: field and groups.

Methods. We used a sample of 167 late-type galaxies with stellar masses of $9 \leq \log \left(M_{\star} / M_{\odot}\right) \leq 12$ drawn from the Calar Alto Legacy Integral Field Area (CALIFA) survey. Firstly, we obtained light- and mass-weighted stellar metallicity profiles and stellar mass density profiles of these galaxies using publicly available data. We then classified them according to their environment into field and group galaxies. Finally, we studied the metallicity of galaxies in these two environments, including a comparison of the metallicity as a function of radius, at a characteristic scale, and as a function of stellar mass surface density. As metallicity depends on galaxy mass, we took special care throughout the study to compare, in all cases, subsamples of galaxies in groups and in the field that have similar masses.

Results. We find significant differences between group and field late-type galaxies in terms of their metallicity: group galaxies are systematically higher in metallicity than their field counterparts. We find that field galaxies, in general, have metallicity profiles that show a negative gradient in their inner regions and a shallower profile at larger radii. This is in contrast to the metallicity profiles of galaxies in groups, which tend to be flat in the inner regions and to have a negative gradient in the outer parts. Regarding the metallicity at the characteristic radius of the luminosity profiles, we consistently find that it is higher for group galaxies irrespective of galaxy mass. At fixed local stellar surface mass density, group galaxies are again higher in metallicity, also the dependence of metallicity on surface density is less important for group galaxies.

Conclusions. The evidence of a clear difference in metallicity between group and field galaxies as a function of mass, spatial scale, and local stellar mass density is indicative of the different evolutionary paths followed by galaxies in groups and in the field. We discuss some possible implications of the observed differences.
\end{abstract}

Key words. galaxies: general - galaxies: stellar content - galaxies: evolution - galaxies: groups: general

\section{Introduction}

The formation and evolution of galaxies is a complicated process that involves the action of different physical mechanisms acting at different temporal and spatial scales. As for their origin, these processes can be due to internal or external, that is, environmental, factors. There are many internal physical mechanisms that can affect the properties of galaxies; for example, supernova (SN) outflows (e.g. Stringer et al. 2012; Bower et al. 2012), feedback from massive stars (e.g. Dalla Vecchia \& Schaye 2008; Hopkins et al. 2012), feedback from active galactic nuclei (AGN; e.g. Nandra et al. 2007; Hasinger 2008; Silverman et al. 2008; Cimatti et al. 2013), halo heating (Marasco et al. 2012), and morphological quenching (Martig et al. 2009). On the other hand, several environmental mechanisms act upon galaxies at different stages of their life. Galaxies in groups and clusters can loose an important fraction of their cold gas because of the pressure of the intracluster hot gas, a process known as ram pressure stripping (e.g. Gunn \& Gott 1972; Abadi et al. 1999; Rasmussen et al. 2006; Jaffé et al. 2012; Hess \& Wilcots 2013). The hot gas can also be removed from the galactic halo, cutting off the supply of gas and consequently stopping star forma- tion. This latter mechanism is known as starvation (e.g. Larson et al. 1980; Bekki 2009; McCarthy et al. 2008; Bahé et al. 2013; Vijayaraghavan \& Ricker 2015). Other mechanisms such as tidal stripping (e.g. Gnedin 2003a; Villalobos et al. 2014) and thermal evaporation (Cowie \& Songaila 1977) could also affect galaxy evolution. Galaxy-galaxy high-speed interactions (e.g. Moore et al. 1996, 1999; Gnedin 2003b) and mergers are mechanisms that can redistribute the gaseous, dark matter, and stellar components of a galaxy, with a resulting change in its properties.

Historically, statistical studies of galaxies have been carried out by analysing their integrated properties, such as star formation, metallicity, colour, magnitudes, and so on. In recent years, thanks to the new generation of integral field spectroscopy (IFS) surveys such as Calar Alto Legacy Integral Field Area (CALIFA; Sánchez et al. 2012a), Sydney-AAO Multi-object Integral field spectrograph (SAMI; Bryant et al. 2015), and MApping Nearby Galaxy at APO (MANGA; Bundy et al. 2015), it has become possible to obtain spatially resolved information on the stellar population in galaxies. These latter instruments have enabled the construction of surveys that include hundreds of galaxies and provide two-dimensional maps for different properties of galaxies, metallicity being among the most studied. Analysis of the 
spatial distribution of metallicity inside galaxies is an important tool to study different physical processes that act at different radii.

Studies of the metallicity distribution in galaxies have been carried out following different approaches and techniques, analysing the gaseous and/or the stellar component. Some of these works have addressed the dependence of metallicity profiles on the environment. However, regardless of the techniques and galaxy samples used, the results continue to be contradictory (Pilyugin \& Grebel 2016), and range from finding no difference within 0.02 dex between field and cluster galaxies (Hughes et al. 2013; Kacprzak et al. 2015) to finding that galaxies in clusters have higher metallicities than those in the field (Shimakawa et al. 2015), or even that star forming (SF) galaxies in clusters are lower in metallicity than those in the field (Valentino et al. 2015). In general, the preferred method to determine the metallicity of SF galaxies is to measure the oxygen abundance $(\mathrm{O} / \mathrm{H})$ in the interstellar medium (ISM) because it is the most abundant heavy element, is easy to detect, and can be used to obtain measurements for a large sample of galaxies through their emission lines. The methods that calibrate abundance relations have been continuously developed and improved over the years thanks to the accumulation of large volumes of increasingly better quality data. However, the different calibrators available to determine the gas-phase metallicity (e.g. Pérez-Montero \& Díaz 2005; Marino et al. 2013; Pérez-Montero 2017) show considerable scatter and it may not be easy to determine whether the variations due to the effects of the environment are greater than this scatter. Many studies (e.g. Ellison et al. 2009), have opted for gas-phase metallicity because stellar metallicity is insensitive to small variations in metallicity. These latter authors find variations of 0.02-0.07 dex between different environments. Stellar metallicity accounts for the chemical enrichment throughout the star formation history (SFH) of the galaxy. Although HII regions contain a "memory" of the SFH (Sánchez et al. 2014), gas-phase metallicity is susceptible to outflows and inflows, among other processes (Wu et al. 2017; Lian et al. 2018a), which can alter the metallicity radial profiles. For this reason, in this paper we focus on stellar metallicity. Although the expected variations are supposed to be small, we are interested in the evolutionary imprint left by processes acting in different environments. These processes may act on shorter timescales in the ISM, but the imprint of their effect, as far as galactic evolution is concerned, will eventually be on the stellar metallicity. It is important to note that, due to the small variations expected from environmental effects, and given the different processes acting at different radii depending on the morphological type and mass of the galaxies, the best way to study these effects is through a spatially resolved analysis; that is, a study of integrated properties, as opposed to IFS, may blur any existing evidence of the acting mechanisms.

Several works that use numerical simulations to study the formation and evolution of discs in galaxies favour an insideout scenario for this component (e.g. Scannapieco et al. 2009; Brook et al. 2012; Tissera et al. 2016) and many observational results using IFS provide support for this. Using the CALIFA survey, Sánchez-Blázquez et al. (2014) find shallow and negative metallicity gradients in disc galaxies. In particular, these latter authors find that luminosity-weighted metallicity gradients are steeper than the mass-weighted ones. They also analyse whether or not the presence of a bar is responsible for the shallow profiles observed, because bars are supposed to produce stellar migrations (e.g. Wielen 1977; Sellwood \& Binney 2002). However, Sánchez-Blázquez et al. (2014) do not find significant differences between barred and non-barred galaxies, in disagreement with the predictions of numerical simulations (e.g. Minchev et al. 2012; Di Matteo et al. 2013; Vincenzo \& Kobayashi 2020). González Delgado et al. (2016a) also find that spiral galaxies have negative metallicity and age gradients, in agreement with an inside-out formation. Further evidence in favour of the inside-out scenario using CALIFA data is presented in García-Benito et al. (2017). They study the mass assembly timescales of 661 CALIFA galaxies that cover wide ranges in both mass and Hubble types. Their results indicate that galaxies form inside-out independently of their stellar mass, stellar mass surface density, or morphology. Lian et al. (2018b), using data from the MaNGA survey, also report negative gradients in both gas and stellar metallicity, the latter being steeper.

Goddard et al. (2017a) use the MANGA survey to study the internal gradients of the stellar population in galaxies. They obtain negative metallicity gradients for both early- and latetype galaxies, and find that gradients are steeper for late-types. In addition, Goddard et al. (2017b) analysed the stellar population properties, age and metallicity, to study the gradients as a function of three characterisations of the environment: local density, tidal strength parameter, and whether a galaxy is central or satellite. In neither case do they find a strong correlation with the environment, and suggest that galaxy mass is the main driver of the stellar population gradients in both early- and latetypes galaxies. Analogously, Zheng et al. (2017) find that the mean age and metallicity gradients are slightly negative. These latter authors conclude that their results are consistent with the inside-out formation scenario. They also study the environmental dependence of age and metallicity at the effective radii, finding that high-mass galaxies are less affected by the environment.

This paper is the second in a series. In the first paper (Coenda et al. 2019), we explore the effects of environment on star formation in late-type galaxies by analysing the radial profiles of the specific star formation rate (sSFR). In that paper, we considered three different environments: field galaxies, galaxies in pairs, and galaxies in groups. Galaxies were selected from CALIFA.

Here, we aim to study the effects of the environment over a longer timescale, analysing stellar metallicity gradients. For this analysis, we use the subsamples of galaxies in groups and in the field selected by Coenda et al. (2019). This paper is organised as follows: in Sect. 2 we provide a detailed description of the CALIFA data that we use, the environment classification, and the metallicity profiles. In Sect. 3, we present our analysis of the metallicity profiles of late-type galaxies in the field and in groups. Finally, we discuss our results in Sect. 4.

\section{The sample}

\subsection{CALIFA}

One of the most important integral field surveys of the last decade considering sample size and the compromise between field of view, spectral coverage, and spatial resolution is the CALIFA survey. In this five-year survey, more than 900 galaxies were observed. The reduced data, ready for scientific exploitation, were made public in three successive releases (DR1, Husemann et al. 2013; DR2, García-Benito et al. 2015; DR3, Sánchez et al. 2016). The instrument used was the Potsdam Multi-Aperture Spectrograph (PMAS, Roth et al. 2005) in the PPaK integral field mode (Kelz et al. 2006) mounted on the $3.5 \mathrm{~m}$ telescope of Calar Alto Observatory. CALIFA observations were performed in two different configurations: on the one hand, using the low-resolution V500 grating covering the wavelength range 
3745-7500 ̊ with a spectral resolution of $6.0 \AA$ (full width at half maximum, FWHM), and on the other using the V1200 grating with a medium spectral resolution of $2.3 \AA$ (FWHM), in the wavelength range $3650-4840 \AA$. The total number of galaxies observed in this second configuration was 484 . From the combination of these two configurations, a third data cube called COMBO is obtained with a spectral resolution of $6.0 \AA$, and a wavelength range of 3700-7500 $\mathrm{A}$. COMBO data cubes for 446 galaxies were made available in DR3.

To construct the CALIFA mother sample, 997 galaxies were selected from the Sloan Digital Sky Survey Data Release 7 (SDSS DR7, Abazajian et al. 2009) photometric galaxy catalogue. With an $r$-band isophotal angular diameter of $45^{\prime \prime}-80^{\prime \prime}$ covering the redshift range $0.005<z<0.03$, the CALIFA mother sample covers the colour-magnitude diagram and probes a wide range of stellar masses, ionisation conditions, and morphological types. The sample morphological classification was performed through visual inspection of the SDSS $r$-band images by five members of the collaboration. Exhaustive information on the sample characterisation can be found in Walcher et al. (2014). More details on the reduction, observation, and data can be found in Husemann et al. (2013), García-Benito et al. (2015), and Sánchez et al. (2016).

de Amorim et al. (2017) analysed all DR3 galaxies with COMBO datacubes using the STARLIGHT ${ }^{1}$ spectral synthesis code (Cid Fernandes et al. 2005). In combination with the organisation code PyCASSO${ }^{2}$ (Cid Fernandes et al. 2013), they built a catalogue of the stellar populations properties of 445 CALIFA galaxies, providing integrated properties and maps of the stellar mass surface density, mean stellar ages and metallicities, stellar dust attenuation, star formation rates, and kinematics. To perform the analysis of star populations, de Amorim et al. (2017) used two sets of single stellar population (SSP) bases called GMe and $\mathrm{CBe}$, similar to the GM and $\mathrm{CB}$ bases presented in Cid Fernandes et al. (2014), but extended in terms of metallicity coverage. As in Coenda et al. (2019), here we use the maps generated with the GMe base which is constructed with a combination of 235 SSP spectra by Vazdekis et al. (2010) for populations over $63 \mathrm{Myr}$ and the models by González Delgado et al. (2005) for younger ages. The initial mass function used is that of Salpeter (1955), and the metallicity covers the seven bins $\left(Z / Z_{\odot}\right)=-2.3,-1.7,-1.3,-0.7,-0.4,0,+0.22$ (Vazdekis et al. 2010) for SSP over $63 \mathrm{Myr}$, and only the four largest metallicities for the younger. The evolutionary tracks are those of Girardi et al. (1993), except for younger ages (1 and $3 \mathrm{Myr}$ ) for which Geneva tracks (Schaller et al. 1992) are used instead.

In the present work we study the effects of environment on the stellar metallicity radial profiles of a sample of late-type galaxies with stellar masses in the range $9 \leq \log \left(M_{\star} / M_{\odot}\right) \leq 12$, where $M_{\star}$ is the total stellar mass obtained from the integrated spectra as indicated in de Amorim et al. (2017). The sample analysed in our work consists in a subsample drawn from the DR3 galaxies for which a COMBO data cube and stellar metallicity maps have been determined.

\subsection{Environments}

The sample of late-type galaxies used in this paper is a subset of the samples used in Coenda et al. (2019), namely those that have available metallicity maps and are either in groups or in the field.

\footnotetext{
1 http://starlight.ufsc.br

2 Python CALIFA Starlight Synthesis organiser, http://pycasso. iaa.es
}

We excluded galaxies in pairs given the small number of them for which stellar metallicity maps are available, which made it meaningless to split them into as many different mass bins as we do with group and field galaxies in the following section. Thus, after excluding those galaxies in pairs, we analyse the metallicity profiles of late-type galaxies in groups and in the field.

The three environments explored in Coenda et al. (2019) are defined in terms of a tracer sample of galaxies from the SDSSDR12 (Alam et al. 2015), with measured redshifts, and restricted to $r$-band Petrosian magnitudes $r \leq 17.77$. Since the spectroscopic sample of the SDSS is incomplete in redshift for galaxies brighter than $r=14.5$, in Coenda et al. (2019) the tracer sample is improved with the inclusion of all galaxies in the DR12 photometric database that have no redshift measured by SDSS, but have available redshift in the $\mathrm{NED}^{3}$ database. As CALIFA only observed nearby galaxies $(z<0.03)$, this addition from the NED database improves the level of completeness of the tracer sample, with the consequent improvement of environment characterisation. We briefly describe how groups and field galaxies are defined in Coenda et al. (2019).

\subsubsection{Galaxies in groups}

Our sample of galaxies in groups includes all CALIFA galaxies that are members of one of the groups of galaxies identified over the tracer sample. Groups of galaxies were identified following Merchán \& Zandivarez (2005). For details of group identification we refer the reader to that paper. Briefly, the group sample was constructed by means of the algorithm developed by Huchra \& Geller (1982), which groups galaxies into systems using a redshift-dependent linking length. This linking length is tuned to retrieve regions with a numerical overdensity of galaxies of 200. A lower limit in membership is imposed, excluding groups with less than four galaxy members. Line-of-sight velocity dispersions are computed using the Gapper estimator in the case of groups with less than 15 members, while for richer groups the bi-weight estimator is used instead (Girardi et al. 1993, 2000). Group virial mass is estimated through the velocity dispersion and the projected virial radius. The resulting sample comprises 17021 groups with at least four members in the redshift range $0<z<0.3$, and their virial masses range from $\sim 1 \times 10^{10} M_{\odot}$ to $\sim 1 \times 10^{16} M_{\odot}$ with a median of $\sim 8.5 \times 10^{13} M_{\odot}$. A total of 204 CALIFA galaxies are found to be in these groups, of which 112 are late type.

\subsubsection{Field galaxies}

We consider as field galaxies those CALIFA galaxies that are not included in the group galaxies defined above and are not likely to be part of a pair of galaxies. We explain briefly now how pairs were defined in Coenda et al. (2019). Firstly, we search among CALIFA galaxies not included in groups for those that are candidates to be in a pair. These are galaxies that have a tracer companion inside a line-of-sight cylinder centred in the CALIFA galaxy and that extends out to a projected radius of $100 \mathrm{kpc}$, and stretches $\pm 1000 \mathrm{~km} \mathrm{~s}^{-1}$ in radial velocity (Alpaslan et al. 2015). This results in a candidate list including 127 galaxies, of which 104 are late type.

3 The NASA/IPAC Extragalactic Database (NED) is operated by the Jet Propulsion Laboratory, California Institute of Technology, under contract with the National Aeronautics and Space Administration, https://ned.ipac.caltech.edu/ 
Secondly, pairs are considered as genuine if they have a lineof-sight relative velocity of the two galaxies smaller than the escape velocity of a suitable dark matter halo (see details in Coenda et al. 2019) at a distance equal to the projected separation of the galaxies (see also Sales et al. 2007). A total of 77 CALIFA galaxies meet this criterion, of which 62 are late type. We note that Barrera-Ballesteros et al. (2015) use a less conservative set of parameters than in the present work for defining pairs from the CALIFA sample.

The remaining CALIFA galaxies that were not classified as being part of either a group or a pair are considered as field galaxies. These amount to 226 galaxies, including 185 that are late type. Some of these galaxies may not be isolated but in actual pairs or groups that our procedure has not been able to detect. Thus, the differences we find in the analyses below between field galaxies and group galaxies could actually be more significant. However, it is unlikely that this possible contamination from galaxies in pairs or groups could change our conclusions.

\subsection{Radial profiles of luminosity and metallicity}

Following Coenda et al. (2019), for the radial profiles determination, we first fit ellipses to the luminosity surface density maps $\left(\mathcal{L}_{5635 \AA}\right)$ provided by de Amorim et al. (2017). These maps were constructed by directly measuring the average flux of the spectra in the spectral window of $(5635 \pm 45) \AA$. Using the task ellipse (Jedrzejewski 1987) within $\mathrm{IRAF}^{4}$ with 1 spaxel step (1") we obtain the ellipses that we later use to obtain the metallicity profiles. de Amorim et al. (2017) made available two sets of metallicity maps (measured in solar units), one weighted by luminosity and the other weighted by mass. We use the ellipses obtained as indicated above as input for a new run of the task ellipse over the metallicity maps. In this way we obtain the metallicity profiles weighted by mass $\left(\log \left(Z_{\mathrm{M}} / Z_{\odot}\right)\right)$ and luminosity $\left(\log \left(Z_{\mathrm{L}} / Z_{\odot}\right)\right)$ that we use to carry out the analyses presented in this paper. We also use the stellar mass surface density, $\Sigma_{\star}$, in units of $M_{\odot} \mathrm{pc}^{-2}$, calculated from the masses derived with STARLIGHT and provided by de Amorim et al. (2017). This quantity measures the mass currently trapped in stars, as it was corrected for the mass that returned to the ISM during stellar evolution. Our final sample of late-type galaxies with radial metallicity maps comprises 60 galaxies in groups and 107 galaxies in the field. Median values of stellar mass are $\log \left(M_{\star} / M_{\odot}\right)=10.72$ and 10.70 for groups and field galaxies, respectively. As a sanity check of the ellipse step considered, we repeated our analysis with a $2^{\prime \prime}$ step instead and found that the median of the radial profiles are not altered substantially. Therefore, all conclusions derived here are maintained.

\section{Results}

We study the effects of external and internal mechanisms on the radial distribution of the metallicity for late-type galaxies. To explore the external effects, we compare late-type galaxies in two discrete environments: field galaxies and galaxies in groups. To analyse the internal processes, for which mass is the main factor, we split our samples of galaxies into five bins of stellar mass: $\log \left(M_{\star} / M_{\odot}\right)=9.00-10.00,10.00-10.50,10.50-10.85$, 10.85-11.20, and 11.20-12.00.

Within each stellar mass bin, the samples of field and group galaxies have, in general, different mass distributions. To avoid

\footnotetext{
4 http://iraf.noao.edu/
}

mass-related biases in our comparison, we construct subsamples of field galaxies (the largest of our samples) randomly selected to have a similar mass distribution within each mass bin to that of the group sample. This procedure was performed 50 times for the field galaxies.

Figure 1 compares radial profiles of light- and massweighted stellar metallicity for galaxies in the field and in groups, split into the five bins of stellar mass mentioned above. The spatial scale is the radius in units of the $r$-band half-light effective radius. This effective radius is computed following Graham et al. (2005), involving the SDSS $r$-band radius that encloses half the Petrosian flux, and the concentration parameter in the same band. We consider the range $0-2.5 r_{\mathrm{e}}$ to stack the radial profiles and we calculate the median value of $r / r_{\mathrm{e}}$ within each interval of size. The subscript $L$ and $M$ correspond to the metallicity weighting by light and mass, respectively. For galaxies in groups, Fig. 1 shows the median of $\log \left(Z_{\mathrm{L}(\mathrm{M})} / Z_{\odot}\right)$ as a function of $r / r_{e}$. Vertical error-bars were computed using the bootstrap re-sampling technique. For galaxies in the field, Fig. 1 shows the mean value of the medians of $\log \left(Z_{\mathrm{L}(\mathrm{M})} / Z_{\odot}\right)$ as a function of $r / r_{e}$, averaged over the 50 random realisations. Error bars in this case are the dispersion around the mean value. Analogously to González Delgado et al. (2016a) and Coenda et al. (2019), we considered only mass bins containing more than five galaxies. We quote in all cases the actual number of galaxies contributing to each profile.

In general, we observe in Fig. 1 that $\log \left(Z_{\mathrm{L}(\mathrm{M})} / Z_{\odot}\right)$ increases with stellar mass, and profiles have negative gradients, as has been reported by other authors (e.g. Sánchez-Blázquez et al. 2014; González Delgado et al. 2016a; Goddard et al. 2017a; Zheng et al. 2017; Lian et al. 2018b). We find that group galaxies are systematically more metallic than their field counterparts. This is found regardless of whether the metallicity is mass- or luminosity-weighted. For the third stellar-mass bin and for both the profiles weighted by mass and by luminosity, we observe that galaxies in groups and in the field have similar stellar metallicities. We observe the same trend for the profiles weighted by mass in the fifth stellar mass bin. However, the differences between galaxies in groups and those in the field are more noticeable in the luminosity weighted metallicity. Therefore, in what follows, we centre our analyses on the luminosity-weighted profiles. For the second, fourth, and fifth mass bins, there is a tendency of the median profiles of the metallicity in groups to have a more flattened metallicity gradient with a negative slope in the outer parts, whereas for field galaxies more linear profiles are observed. This results in field and group galaxies having relatively similar metallicities at both extremes, that is, in the innermost and outermost regions. However, the first mass bin shows a different behaviour. Although in this case, we also observe that group galaxies have higher metallicity than galaxies in the field, the metallicity profile of the former shows a different shape compared to the other mass bins. In this bin, the metallicity profile of group galaxies presents a convex shape. As a consequence, the differences between the outer and inner radial zones are maximum, and in the middle, the stellar metallicities of both group and field galaxies are similar. Galaxies in this mass bin have the lowest surface brightnesses. Although we verified that all galaxies in the bin contribute to the whole range of $r / r_{e}$ probed, caution should be taken regarding the behaviour in the outermost parts because of a possible effect of low signal-to-noise ratio.

If we assume that field galaxies have evolved virtually in isolation, or at least that they have been exposed to fewer environmental effects than group galaxies, the differences we observe in 
$9.00 \leq \log \left(\mathrm{M}_{\star}\right) \leq 10.0010 .00 \leq \log \left(\mathrm{M}_{\star}\right) \leq 10.5010 .50 \leq \log \left(\mathrm{M}_{\star}\right) \leq 10.8510 .85 \leq \log \left(\mathrm{M}_{\star}\right) \leq 11.2011 .20 \leq \log \left(\mathrm{M}_{\star}\right) \leq 12.00$
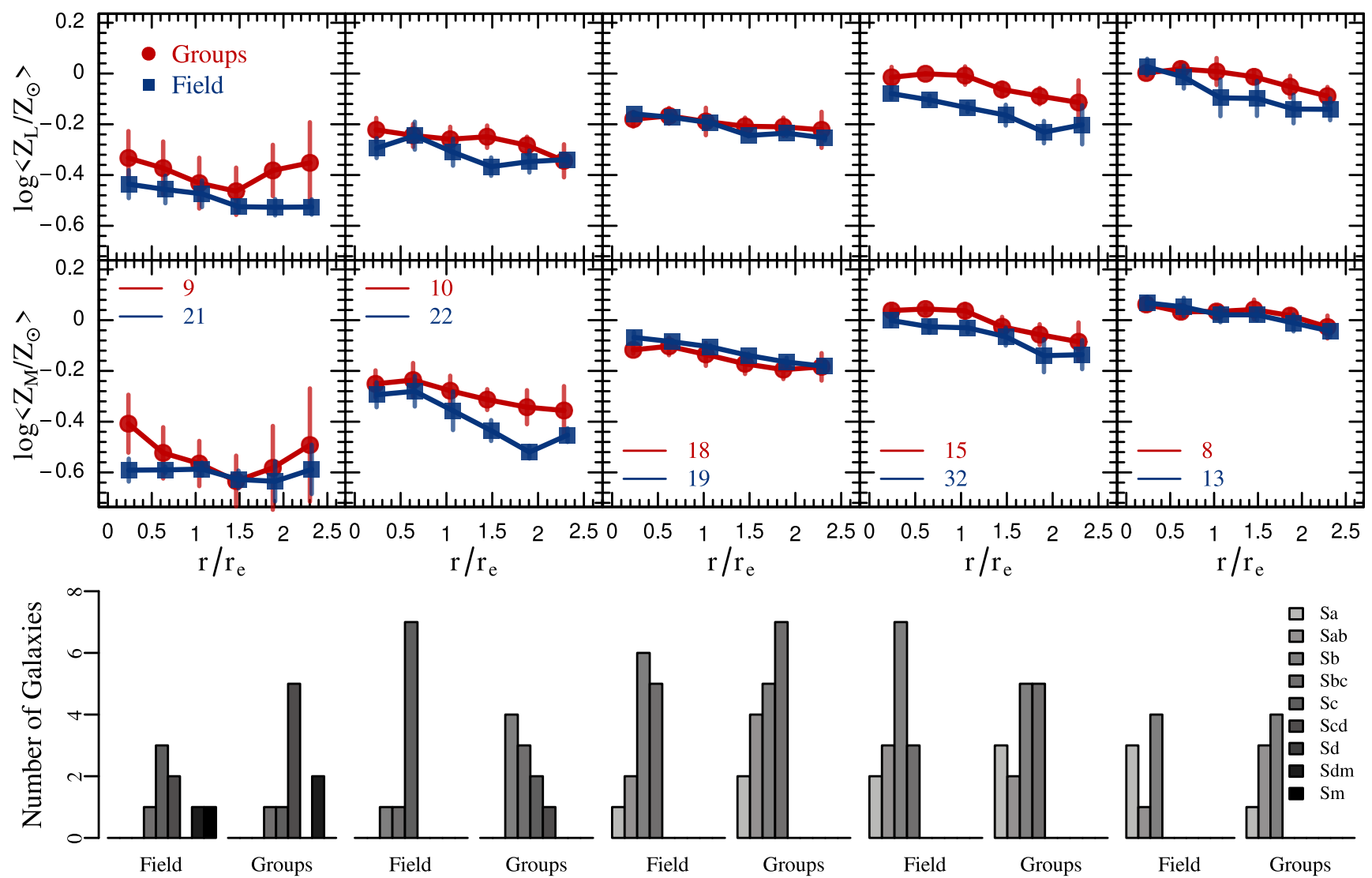

Fig. 1. Stacked profile of the metallicity weighted by light (top panel) and mass (central panel) for late-type galaxies, scaled to the $r$-band halflight effective radius, as a function of the mass and the environment. Galaxies in groups are shown as red dots and lines, and field galaxies as blue dots and lines. Red symbols represent the median in each radial size bin for galaxies in groups. Vertical error bars were computed using the bootstrap re-sampling technique. The blue symbols show the mean value and its dispersion of the field galaxies for 50 randoms runs. Bottom panel: distribution of Hubble type as a function of environment and stellar mass. For field galaxies, we show the mean distribution for 50 randoms runs performed.

the metallicity profiles can be related directly to environmental action.

The bottom panel of Fig. 1 shows the distribution of Hubble type as a function of stellar mass and environment for our sample of late-type galaxies. For field galaxies, we show the average distribution of the 50 random runs performed. We observe that the morphology distributions are relatively similar in both environments for each stellar mass bin. The only probable exception is the second mass bin, where we observe a tendency of group galaxies to have earlier morphologies than field galaxies. This characteristic of the sample may be partially responsible for the observed differences in metallicity between the two environments. It should be noted that this is the bin of mass that presents the greatest difference in metallicity. We also note that each stellar mass bin implies a different set of morphologies. As stellar mass increases, a higher fraction of $\mathrm{Sa}$ and $\mathrm{Sb}$ galaxies is observed. It is worth noting that the mass-dependent morphological mixing in our samples implies that we are not probing a unique galaxy class across the stellar mass range. On the contrary, galaxies in each bin constitute a completely independent sample, and the only common feature bin-to-bin is that galaxies are late type.

In Coenda et al. (2019) we explore whether AGN feedback or the presence of a bar play a role in shaping SSFR profiles. In contrast to SFR, which involves short timescales, in this work we study the profiles of the stellar metallicities, where different physical mechanisms have acted at different temporal and spatial scales throughout the lifetime of the galaxies. The size of our sample does not allow for a separate analysis of whether AGNs or bars can play a role in the observed stellar metallicity. However, we observe that the mere presence of an AGN at $z=0$ does not necessarily imply that it has played a role in shaping the observed galaxy stellar metallicity. An AGN can be a transient phenomenon in a galaxy. With AGN duty cycles spanning timescales in the range $10^{6}-10^{8}$ yr (Haehnelt \& Rees 1993; Davis et al. 2014; Storchi-Bergmann \& Schnorr-Müller 2019), we expect that the AGN effects on stellar metallicity should be due to past and not current AGN activity. Regarding bars, there is no consensus in the literature as to the longevity of bars, nor how many bar events the average galaxy experiences during its lifetime (Sellwood 1999; Athanassoula 2002; Bournaud \& Combes 2002; Elmegreen et al. 2004; Combes 2004; Regan \& Teuben 2004; Pérez et al. 2008; James \& Percival 2016). While several authors have observed a correlation between gas abundance gradients and the presence of bars, in particular, a flattening of the gradient (Vila-Costas \& Edmunds 1992; Martin \& Roy 1994; Zaritsky et al. 1994), more recent works have found no evidence of such a correlation by analysing both gas-phase metallicity 

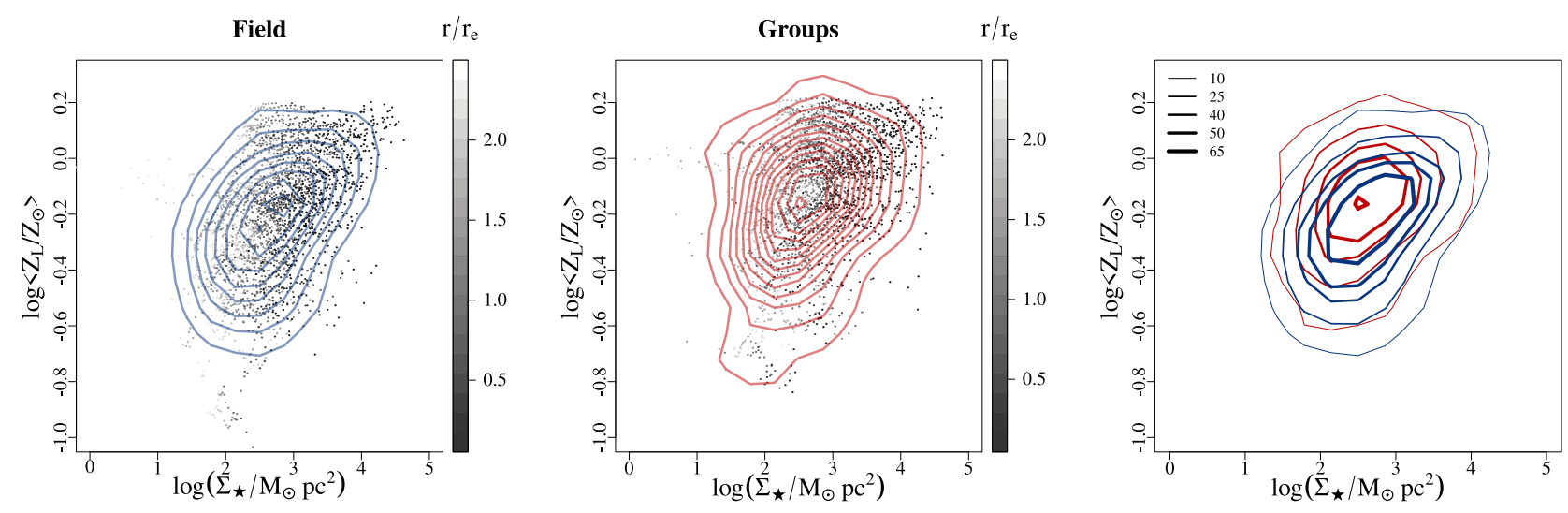

$9.00 \leq \log \left(\mathrm{M}_{\star}\right) \leq 10.00 \quad 10.00 \leq \log \left(\mathrm{M}_{\star}\right) \leq 10.50 \quad 10.50 \leq \log \left(\mathrm{M}_{\star}\right) \leq 10.85 \quad 10.85 \leq \log \left(\mathrm{M}_{\star}\right) \leq 11.2011 .20 \leq \log \left(\mathrm{M}_{\star}\right) \leq 12.00$

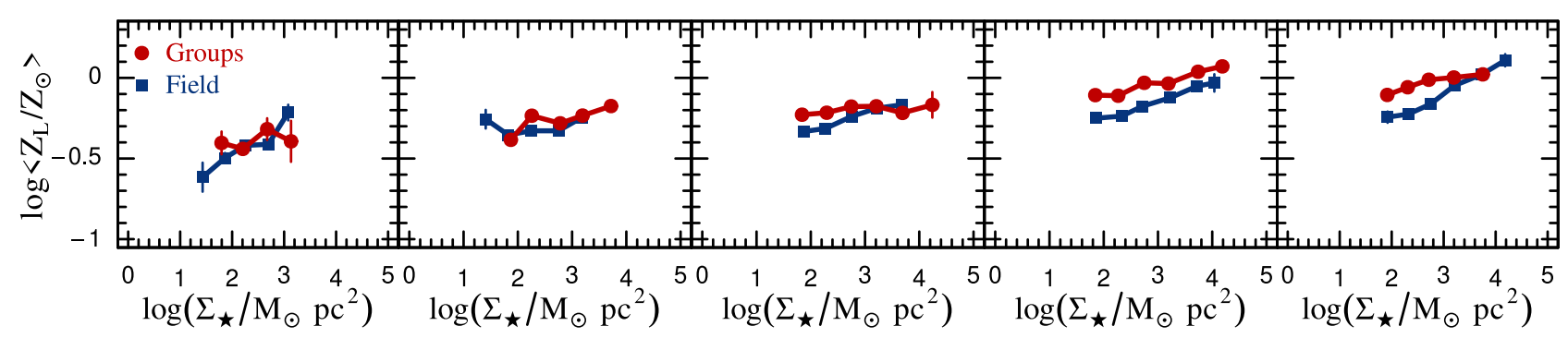

Fig. 2. Top panels: light-weighted metallicity as a function of stellar mass surface density $\Sigma_{\star}$. Dots are colour-coded in tones of grey according to their distance $r / r_{e}$. The left panel corresponds to galaxies in the field, the central panel shows galaxies in groups, and the right panel shows the iso-contour levels for galaxies in groups (red lines) and galaxies in the field (blue lines). The bottom panels show the median value of $\log \left(Z_{L} / Z_{\odot}\right)$ as a function of $\Sigma_{\star}$ for the five stellar-mass bins considered. Vertical error bars were computed using the bootstrap re-sampling technique.

and stellar metallicity (Sánchez et al. 2012b, 2014; SánchezBlázquez et al. 2014; Cheung et al. 2015; Sánchez-Menguiano et al. 2016).

The upper panels of Fig. 2 show the light-weighted metallicity as a function of stellar mass surface density $\log \left(\Sigma_{\star}\right)$ for galaxies in the field and in groups. Field galaxies shown in Fig. 2 are a random realisation of 107 galaxies from the field sample, which were selected to have the same overall mass distribution as the group sample. Each galaxy, in either of the two samples, contributes several points to this figure. The number of points varies from galaxy to galaxy, as does the number of radial bins that each profile has. Points are colour-coded in tones of grey according to their radial distance in terms of the effective radius, $r / r_{\mathrm{e}}$. The solid curves show the number-density isocontour levels. The stellar mass surface density and metallicity are strongly correlated although we observe significant scatter. To compare galaxies in the field and groups, the upper right panel of Fig. 2 shows the iso-contour for each environment considered. We clearly observe that galaxies in groups are more metallic for a fixed value of stellar mass surface density.

For a better comparison between group and field galaxies, we split both samples into the five mass bins used in Fig. 1 and computed the median value of the $\log \left(Z_{\mathrm{L}} / Z_{\odot}\right)$ as a function of the stellar mass surface density, $\log \left(\Sigma_{\star}\right)$. This is shown in the bottom panels of Fig. 2. Clearly, metallicity depends on both mass and stellar mass surface density, that is, a global and a local property, respectively. Mass is a major source of scatter in the top panels of this figure. We observe no clear distinction between field and group galaxies in the two lowest mass bins. From the third bin onward, we observe that group galaxies tend to be more metallic at low to intermediate $\log \left(\Sigma_{\star}\right)$ values, although it is not clear whether or not the differences are significant in the third stellar mass bin. Another interesting feature in this figure is that the metallicity of field galaxies tends to depend more strongly on stellar mass surface density than that of galaxies in groups.

A strong correlation between stellar metallicity and the stellar mass surface density was reported by Rosales-Ortega et al. (2012), Sánchez et al. (2013), and González Delgado et al. (2014). This correlation can be considered as a local process acting in galaxies. Previously, Bell \& de Jong (2000) analysed spiral galaxies and found that the stellar mass surface density of galaxies drives their SFH, and that $M_{\star}$ is a less important parameter. These previous works argue that stellar metallicities are mainly governed by the stellar mass surface density in disc galaxies and by the total mass in spheroids. Our results suggest that both stellar mass surface density and the integrated stellar mass impact the SFH of late-types galaxies. Moreover, we also find that the environment plays an important role in modelling the metallicity profiles.

A complementary quantity from our profiles is the metallicity value at $r_{e}$. Figure 3 shows the median light-weighted stellar metallicity at $r_{e}$ as a function of the stellar mass of the galaxy. The metallicity at $r_{e}$ is correlated with $M_{\star}$ for late-type galaxies, and this correlation depends on the environment. Low-mass galaxies have lower metallicity than high-mass galaxies. Again, galaxies in groups show higher metallicity than galaxies in the field for a fixed value of stellar mass. Zheng et al. (2017) find that low-mass galaxies tend to have lower metallicity in lowdensity environments while high-mass galaxies are less affected by environment. Our results suggest that the environment affects metallicities in the whole range of stellar mass, however future analyses with larger samples are necessary to confirm this. 


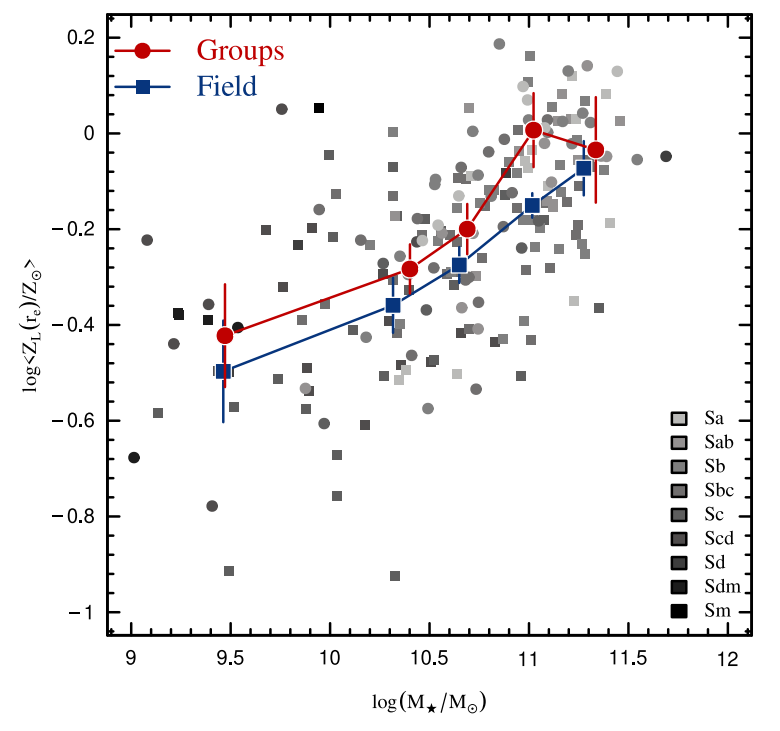

Fig. 3. Light-weighted metallicity at $r_{\mathrm{e}}$ as a function of stellar mass. Dots are colour-coded in tones of grey according to their Hubble type. The lines and dots show the median values of $\log \left(Z_{L}\left(r_{e}\right) / Z_{\odot}\right)$ for galaxies in groups (red) and galaxies in the field (blue).

\section{Discussion and conclusions}

In this paper we present a comparative analysis of the stellar metallicity profiles of late-type galaxies in the field and in groups using publicly available CALIFA data. We focus on three comparative analyses of the metallicity of late-type galaxies: (i) the metallicity profiles in five stellar mass bins, (ii) the relation between metallicity and stellar mass surface density, and (iii) the metallicity at the effective radius. Thus, our analyses compare the metallicity of galaxies as a function of scale (i), at a characteristic scale (iii), and as a function of a local properties (ii). As most galaxy properties, including metallicity, depend on galaxy mass, we take special care throughout the paper in order to compare, in all cases, subsamples of galaxies in groups and in the field that have similar mass distributions within the mass ranges analysed, however broad or thin these ranges might be. In all cases we find significant differences between group and field late-type galaxies. Our results are in contrast to those of Goddard et al. (2017b), who find that stellar population gradients show no significant correlation with galaxy environment regardless of the different characterisations of environment they use. This difference between our results and those of Goddard et al. (2017b) could be due to our choice of splitting galaxies into two discrete environments.

Regarding the comparison of the radial profiles of metallicity, we find that field galaxies in general have metallicity profiles that show a negative gradient in their inner regions and a shallower profile at larger radii. This is in contrast to the metallicity profiles of group galaxies, which tend to be flat in the inner regions and show a negative gradient in the outer parts. As bulges of late-type galaxies are denser and have older stars than the disc, they are expected to be more metallic than the outer parts of the galaxy.

A plausible scenario could be one in which SN ejections throughout the lifetime of a galaxy are accreted back into the disc in field galaxies, thus increasing the metallicity of stars formed later in the outer parts of the disc. This should be less efficient in groups because of environmental effects such as rampressure stripping or strangulation. Group galaxies have higher metallicity than field galaxies at most scales, and notably at the characteristic radius. Since groups are dense environments, galaxies in groups should, on average, have been formed earlier (i.e. downsizing), thus having more time to produce metals. On the other hand, mergers are common in groups, and they tend to redistribute metals within galaxies. Furthermore, mergers could add metals to galaxies by the accretion of earlier-type satellite galaxies.

Analysing the light-weighted stellar metallicity at $r_{e}$, we consistently find that the correlation depends on the environment. Again, galaxies in groups show a higher value of metallicity than galaxies in the field at fixed stellar mass. These results are consistent with the findings of Zheng et al. (2017); however, in our case the evidence is not only present for low-mass galaxies, but in nearly the entire mass range, the only exception being the highest mass bin, where the median values of metallicity are indistinguishable.

Our analysis of the dependence of metallicity on stellar mass surface density shows that, in general, at fixed local density, group galaxies have higher metallicity, which backs up the idea that they formed earlier. Another general trend is that the dependence of metallicity on surface density is less important in group galaxies, which may be indicative of a more effective mix, which can be thought of in terms of more frequent mergers throughout their lifetimes.

As seen in previous works, $\Sigma_{\star}$ is a good tracer of the local star population properties where both age and metallicity correlate with $\Sigma_{\star}$ (Rosales-Ortega et al. 2012; Sánchez et al. 2013; González Delgado et al. 2014). In particular, in discs, $\Sigma_{\star}$ would regulate the mean stellar ages and metallicities, while in spheroids, both in spiral bulges and elliptical galaxies, $\Sigma_{\star}$ would play a minor role. This is due, as mentioned above, to the fact that in spheroids the chemical enrichment occurred faster and at an earlier stage than in discs, in full agreement with the inside-out scenario (Pérez et al. 2013; González Delgado et al. 2014, 2015; Sánchez-Blázquez et al. 2014; Sánchez et al. 2014; García-Benito et al. 2017). This would imply that stellar metallicity is governed by local processes in discs and by global processes in spheroids (González Delgado et al. 2016b). Our $\Sigma_{\star}$ analysis shows how, from medium to high densities (which we could associate with spheroids), the effect of the environment is diluted, while the major differences between group and field galaxies occur as $\Sigma_{\star}$ decreases. This effect is evidently more noticeable in the medium to high mass bins, that is, bins in which there is a more significant presence of spheroids because of the morphological distribution of the sample. This could therefore indicate that the environment plays an important role in the chemical evolution of discs and perhaps a minor role in spheroids.

Alongside strong evidence of the universality of the insideout formation of galaxies, García-Benito et al. (2017) showed a complex multivariate dependence of the mass assembly on stellar mass, stellar mass surface density, and Hubble type. In Coenda et al. (2019) and here, we have taken special care of these factors in our analyses, and have shown that environment is another important factor in galaxy formation and should be taken into account. In this paper in particular, we present evidence of a clear difference in metallicity between group and field galaxies as a function of mass, spatial scale, and local stellar mass density. From an earlier start, a greater number of mergers experienced during their lifetimes, and the action of other environmental mechanisms, it is clear that late-type galaxies in groups have followed a different evolutionary path compared to their field counterparts. 
Acknowledgements. This paper is based on data obtained by the CALIFA survey (http://califa.caha.es) which is based on observations collected at the Centro Astronómico Hispano Alemán (CAHA) at Calar Alto, operated jointly by the Max-Planck-Institut für Astronomie and the Instituto de Astrofísica de Andalucía (CSIC). This research has made use of the NASA/IPAC Extragalactic Database (NED), which is operated by the Jet Propulsion Laboratory, California Institute of Technology, under contract with the National Aeronautics and Space Administration. This paper has been partially supported with grants from Consejo Nacional de Investigaciones Científicas y Técnicas (PIP 11220170100548CO) Argentina, Fondo para la Investigación Científica y Tecnológica (FonCyT, PICT-2017-3301), and Secretaría de Ciencia y Tecnología Universidad Nacional de Córdoba, Argentina.

\section{References}

Abadi, M. G., Moore, B., \& Bower, R. G. 1999, MNRAS, 308, 947

Abazajian, K. N., Adelman-McCarthy, J. K., Agüeros, M. A., et al. 2009, ApJS, 182,543

Alam, S., Albareti, F. D., Allende Prieto, C., et al. 2015, ApJS, 219, 12

Alpaslan, M., Driver, S., Robotham, A. S. G., et al. 2015, MNRAS, 451, 3249

Athanassoula, E. 2002, Disks of Galaxies: Kinematics, Dynamics and Peturbations, eds. E. Athanassoula, A. Bosma, \& R. Mujica, ASP Conf. Ser. 275, 141

Bahé, Y. M., McCarthy, I. G., Balogh, M. L., \& Font, A. S. 2013, MNRAS, 430, 3017

Barrera-Ballesteros, J. K., García-Lorenzo, B., Falcón-Barroso, J., et al. 2015, A\&A, 582, A21

Bekki, K. 2009, MNRAS, 399, 2221

Bell, E. F., \& de Jong, R. S. 2000, MNRAS, 312, 497

Bournaud, F., \& Combes, F. 2002, A\&A, 392, 83

Bower, R. G., Benson, A. J., \& Crain, R. A. 2012, MNRAS, 422, 2816

Brook, C. B., Stinson, G. S., Gibson, B. K., et al. 2012, MNRAS, 426, 690

Bryant, J. J., Owers, M. S., Robotham, A. S. G., et al. 2015, MNRAS, 447, 2857

Bundy, K., Bershady, M. A., Law, D. R., et al. 2015, ApJ, 798, 7

Cheung, E., Conroy, C., Athanassoula, E., et al. 2015, ApJ, 807, 36

Cid Fernandes, R., Mateus, A., Sodré, L., Stasińska, G., \& Gomes, J. M. 2005 MNRAS, 358, 363

Cid Fernandes, R., Pérez, E., García Benito, R., et al. 2013, A\&A, 557, A86

Cid Fernandes, R., González Delgado, R. M., García Benito, R., et al. 2014, A\&A, 561, A130

Cimatti, A., Brusa, M., Talia, M., et al. 2013, ApJ, 779, L13

Coenda, V., Mast, D., Martínez, H. J., Muriel, H., \& Merchán, M. E. 2019, A\&A, 621, A98

Combes, F. 2004, The Interplay Among Black Holes Stars and ISM in Galactic Nuclei, eds. T. Storchi-Bergmann, L. C. Ho, \& H. R. Schmitt, IAU Symp., 222,383

Cowie, L. L., \& Songaila, A. 1977, Nature, 266, 501

Dalla Vecchia, C., \& Schaye, J. 2008, MNRAS, 387, 1431

Davis, B. L., Berrier, J. C., Johns, L., et al. 2014, ApJ, 789, 124

de Amorim, A. L., García-Benito, R., Cid Fernandes, R., et al. 2017, MNRAS, 471,3727

Di Matteo, P., Haywood, M., Combes, F., Semelin, B., \& Snaith, O. N. 2013 A\&A, 553, A102

Ellison, S. L., Simard, L., Cowan, N. B., et al. 2009, MNRAS, 396, 1257

Elmegreen, B. G., Elmegreen, D. M., \& Hirst, A. C. 2004, ApJ, 612, 191

García-Benito, R., Zibetti, S., Sánchez, S. F., et al. 2015, A\&A, 576, A135

García-Benito, R., González Delgado, R. M., Pérez, E., et al. 2017, A\&A, 608, A27

Girardi, L., Bressan, A., Bertelli, G., \& Chiosi, C. 2000, A\&AS, 141, 371

Girardi, M., Biviano, A., Giuricin, G., Mardirossian, F., \& Mezzetti, M. 1993, ApJ, 404, 38

Gnedin, O. Y. 2003a, ApJ, 582, 141

Gnedin, O. Y. 2003b, ApJ, 589, 752

Goddard, D., Thomas, D., Maraston, C., et al. 2017a, MNRAS, 466, 473

Goddard, D., Thomas, D., Maraston, C., et al. 2017b, MNRAS, 465, 688

González Delgado, R. M., Cerviño, M., Martins, L. P., Leitherer, C., \& Hauschildt, P. H. 2005, MNRAS, 357, 945

González Delgado, R. M., Cid Fernandes, R., García-Benito, R., et al. 2014, ApJ, 791, L16

González Delgado, R. M., García-Benito, R., Pérez, E., et al. 2015, A\&A, 581, A103

González Delgado, R. M., Cid Fernandes, R., Pérez, E., et al. 2016a, A\&A, 590, A44

González Delgado, R. M., Cid Fernandes, R., Pérez, E., et al. 2016b, A\&A, 590, A44

Graham, A. W., Driver, S. P., Petrosian, V., et al. 2005, AJ, 130, 1535
Gunn, J. E., \& Gott, J. R. I. 1972, ApJ, 176,

Haehnelt, M. G., \& Rees, M. J. 1993, MNRAS, 263, 168

Hasinger, G. 2008, A\&A, 490, 905

Hess, K. M., \& Wilcots, E. M. 2013, AJ, 146, 124

Hopkins, P. F., Quataert, E., \& Murray, N. 2012, MNRAS, 421, 3522

Huchra, J. P., \& Geller, M. J. 1982, ApJ, 257, 423

Hughes, T. M., Cortese, L., Boselli, A., Gavazzi, G., \& Davies, J. I. 2013, A\&A, 550, A115

Husemann, B., Jahnke, K., Sánchez, S. F., et al. 2013, A\&A, 549, A87

Jaffé, Y. L., Poggianti, B. M., Verheijen, M. A. W., Deshev, B. Z., \& van Gorkom,

J. H. 2012, ApJ, 756, L28

James, P. A., \& Percival, S. M. 2016, MNRAS, 457, 917

Jedrzejewski, R. I. 1987, MNRAS, 226, 747

Kacprzak, G. G., Yuan, T., Nanayakkara, T., et al. 2015, ApJ, 802, L26

Kelz, A., Verheijen, M. A. W., Roth, M. M., et al. 2006, PASP, 118, 129

Larson, R. B., Tinsley, B. M., \& Caldwell, C. N. 1980, ApJ, 237, 692

Lian, J., Thomas, D., Maraston, C., et al. 2018a, MNRAS, 474, 1143

Lian, J., Thomas, D., Maraston, C., et al. 2018b, MNRAS, 476, 3883

Marasco, A., Fraternali, F., \& Binney, J. J. 2012, MNRAS, 419, 1107

Marino, R. A., Rosales-Ortega, F. F., Sánchez, S. F., et al. 2013, A\&A, 559, A114

Martig, M., Bournaud, F., Teyssier, R., \& Dekel, A. 2009, ApJ, 707, 250

Martin, P., \& Roy, J.-R. 1994, ApJ, 424, 599

McCarthy, I. G., Frenk, C. S., Font, A. S., et al. 2008, MNRAS, 383, 593

Merchán, M. E., \& Zandivarez, A. 2005, ApJ, 630, 759

Minchev, I., Famaey, B., Quillen, A. C., et al. 2012, A\&A, 548, A127

Moore, B., Katz, N., Lake, G., Dressler, A., \& Oemler, A. 1996, Nature, 379, 613

Moore, B., Lake, G., Quinn, T., \& Stadel, J. 1999, MNRAS, 304, 465

Nandra, K., Georgakakis, A., Willmer, C. N. A., et al. 2007, ApJ, 660, L11

Pérez-Montero, E. 2017, PASP, 129, 043001

Pérez-Montero, E., \& Díaz, A. I. 2005, MNRAS, 361, 1063

Pérez, I., Sánchez-Blázquez, P., \& Zurita, A. 2008, Formation and Evolution of Galaxy Disks, eds. J. G. Funes, \& E. M. Corsini, ASP Conf. Ser., 396, 375

Pérez, E., Cid Fernandes, R., González Delgado, R. M., et al. 2013, ApJ, 764, L1 Pilyugin, L. S., \& Grebel, E. K. 2016, MNRAS, 457, 3678

Rasmussen, J., Ponman, T. J., \& Mulchaey, J. S. 2006, MNRAS, 370, 453

Regan, M. W., \& Teuben, P. J. 2004, ApJ, 600, 595

Rosales-Ortega, F. F., Sánchez, S. F., Iglesias-Páramo, J., et al. 2012, ApJ, 756, L31

Roth, M. M., Kelz, A., Fechner, T., et al. 2005, PASP, 117, 620

Sales, L. V., Navarro, J. F., Abadi, M. G., \& Steinmetz, M. 2007, MNRAS, 379, 1475

Salpeter, E. E. 1955, ApJ, 121, 161

Sánchez, S. F., Kennicutt, R. C., \& Gil de Paz, A. 2012a, A\&A, 538, A8

Sánchez, S. F., Rosales-Ortega, F. F., Marino, R. A., et al. 2012b, A\&A, 546, A2

Sánchez, S. F., Rosales-Ortega, F. F., Jungwiert, B., et al. 2013, A\&A, 554, A58

Sánchez, S. F., Rosales-Ortega, F. F., Iglesias-Páramo, J., et al. 2014, A\&A, 563, A49

Sánchez, S. F., García-Benito, R., Zibetti, S., et al. 2016, A\&A, 594, A36

Sánchez-Blázquez, P., Rosales-Ortega, F. F., Méndez-Abreu, J., et al. 2014, A\&A, 570, A6

Sánchez-Menguiano, L., Sánchez, S. F., Pérez, I., et al. 2016, A\&A, 587, A70

Scannapieco, C., White, S. D. M., Springel, V., \& Tissera, P. B. 2009, MNRAS, 396, 696

Schaller, G., Schaerer, D., Meynet, G., \& Maeder, A. 1992, A\&AS, 96, 269

Sellwood, J. A. 1999, Galaxy Dynamics - A Rutgers Symposium, eds. D. R. Merritt, M. Valluri, \& J. A. Sellwood, ASP Conf. Ser., 182, 96

Sellwood, J. A., \& Binney, J. J. 2002, MNRAS, 336, 785

Shimakawa, R., Kodama, T., Tadaki, K.-I., et al. 2015, MNRAS, 448, 666

Silverman, J. D., Mainieri, V., Lehmer, B. D., et al. 2008, ApJ, 675, 1025

Storchi-Bergmann, T., \& Schnorr-Müller, A. 2019, Nat. Astron., 3, 48

Stringer, M. J., Bower, R. G., Cole, S., Frenk, C. S., \& Theuns, T. 2012, MNRAS, 423, 1596

Tissera, P. B., Machado, R. E. G., Sanchez-Blazquez, P., et al. 2016, A\&A, 592, A93

Valentino, F., Daddi, E., Strazzullo, V., et al. 2015, ApJ, 801, 132

Vazdekis, A., Sánchez-Blázquez, P., Falcón-Barroso, J., et al. 2010, MNRAS, 404,1639

Vijayaraghavan, R., \& Ricker, P. M. 2015, MNRAS, 449, 2312

Vila-Costas, M. B., \& Edmunds, M. G. 1992, MNRAS, 259, 12

Villalobos, A., De Lucia, G., \& Murante, G. 2014, MNRAS, 444, 313

Vincenzo, F., \& Kobayashi, C. 2020, MNRAS, 496, 80

Walcher, C. J., Wisotzki, L., Bekeraité, S., et al. 2014, A\&A, 569, A1

Wielen, R. 1977, A\&A, 60, 263

Wu, P.-F., Zahid, H. J., Hwang, H. S., \& Geller, M. J. 2017, MNRAS, 468, 1881

Zaritsky, D., Kennicutt, J., Robert, C., \& Huchra, J.P. 1994, ApJ, 420, 87

Zheng, Z., Wang, H., Ge, J., et al. 2017, MNRAS, 465, 4572 